\title{
RIEMANN-HILBERT PROBLEM FOR FIRST-ORDER ELLIPTIC SYSTEMS WITH CONSTANT LEADING COEFFICIENTS ON THE PLANE
}

\section{A. P. Soldatov and O. V. Chernova}

UDC 517.9

Abstract. In a finite domain $D$ of the complex plane bounded by a smooth contour $\Gamma$, we consider the Riemann-Hilbert boundary-value problem $\operatorname{Re} C U^{+}=f$ for the first-order elliptic system

$$
\frac{\partial U}{\partial y}-A \frac{\partial U}{\partial x}+a(z) U(z)+b(z) \overline{U(z)}=F(z)
$$

with constant leading coefficients. Here + means the boundary value of the function $U$ on $\Gamma$, the constant matrices $A_{1}, A_{2} \in \mathbb{C}^{l \times l}$ and the $(l \times l)$-matrix coefficients $a$ and $b$ belong to the Hölder class $C^{\mu}, 0<\mu<1$, and $(l \times l)$-matrix function $C$ belongs to the class $C^{\mu}(\Gamma)$. We prove that in the class $U \in C^{\mu}(\bar{D}) \cap C^{1}(D)$, this problem is a Fredholm problem and its index is given by the formula

$$
\varkappa=-\sum_{j=1}^{m} \frac{1}{\pi}[\arg \operatorname{det} G]_{\Gamma_{j}}+(2-m) l .
$$

Keywords and phrases: elliptic systems, Riemann-Hilbert problem, index formula, Fredholm operator.

AMS Subject Classification: 35Jxx, 58J10, 58J20

In a finite domain $D$ of the complex plane bounded by a smooth contour $\Gamma$ we consider the first-order elliptic system

$$
A_{1} \frac{\partial U}{\partial x}+A_{2} \frac{\partial U}{\partial x}+a(z) U(z)+b(z) \overline{U(z)}=F(z), \quad z \in D
$$

where the constant matrices $A_{1}, A_{2} \in \mathbb{C}^{l \times l}$ and $(l \times l)$-matrix coefficients $a$ and $b$ belong to the Hölder class $C^{\mu}(D), 0<\mu<1$. The ellipticity condition is as follows: both matrices $A_{j}$ are invertible and the matrix $A=-A_{2}^{-1} A_{1}$ has no real eigenvalues. Multiplying the system by $A_{2}^{-1}$ and introducing new notation, we can write it in the form

$$
\frac{\partial U}{\partial y}-A \frac{\partial U}{\partial x}+a(z) U(z)+b(z) \overline{U(z)}=F(z) .
$$

It is convenient to represent the set of eigenvalues of the matrix $A$ as the union $\sigma_{1} \cup \bar{\sigma}_{2}$, where both sets $\sigma_{1}$ and $\sigma_{2}$ lie in the upper half-plane $\operatorname{Im} \nu>0$ and $\bar{\sigma}_{2}=\left\{\bar{\nu}, \nu \in \sigma_{2}\right\}$. Then the matrix $A$ can be reduced to the following Jordan form:

$$
\widetilde{B}^{-1} A \widetilde{B}=\widetilde{J}, \quad \widetilde{J}=\operatorname{diag}\left(J_{1}, \bar{J}_{2}\right),
$$

where $J_{k} \in \mathbb{C}^{l_{k} \times l_{k}}, k=1,2$, consists of Jordan cells with eigenvalues $\nu \in \sigma_{k}$. Surely, $l=l_{1}+l_{2}$ and the cases $l_{1}=0$ or $l_{2}=0$, when one of the sets $\sigma_{k}$ is empty, are not excluded. Thus, in this representation the eigenvalues of the matrix $J_{k}$ form the set $\sigma_{k}$. According to (2), we represent the matrix $\widetilde{B}$ in the block form

$$
\widetilde{B}=\left(B_{1}, \bar{B}_{2}\right), \quad B_{k} \in \mathbb{C}^{l \times l_{k}} .
$$

For a given $(l \times l)$-matrix-valued function $C \in C^{\mu}(\Gamma)$, we consider the Riemann-Hilbert boundaryvalue problem for the system (1):

$$
\operatorname{Re} C U^{+}=f
$$

Translated from Itogi Nauki i Tekhniki, Seriya Sovremennaya Matematika i Ee Prilozheniya. Tematicheskie Obzory, Vol. 149, Proceedings of the International Conference "Actual Problems of Applied Mathematics and Physics," Kabardino-Balkaria, Nalchik, May 17-21, 2017, 2018. 
where + means the boundary value of the function $U$ on $\Gamma$. Assuming that $F \in C^{\mu}(\bar{D})$ and $f \in C^{\mu}(\Gamma)$, it is natural to consider this problem in the class of classical solutions $C^{\mu}(\bar{D}) \cap C^{1}(D)$ of the system $(1)$.

In the space of right-hand sides $(F, f) \in C^{\mu}(\bar{D}) \times C^{\mu}(\Gamma)$, we introduce the bilinear form

$$
\langle(F, f),(\widetilde{F}, \widetilde{f})\rangle=\int_{D} \operatorname{Re}[F(z) \widetilde{F}(z)] d_{2} z+\int_{\Gamma} f(t) \widetilde{f}(t) d_{1} t,
$$

where $d_{2} z$ and $d_{1} t$ mean the area and arclength elements, respectively, and the notation $F \widetilde{F}$ for two vectors $F=\left(F_{1}, \ldots, F_{l}\right)$ and $\widetilde{F}=\left(\widetilde{F}_{1}, \ldots, \widetilde{F}_{l}\right)$ means their scalar product $F_{1} \widetilde{F}_{1}+\ldots+F_{l} \widetilde{F}_{l}$; the notation $f \tilde{f}$ has a similar sense.

The main result of this paper consists of establishing a criterion for the Fredholm property of the problem (1), (4) in the class $U \in C^{\mu}(\bar{D}) \cap C^{1}(D)$ and the formula of its index. The Fredholm property is understood in the following sense: the space $X$ of solutions of the homogeneous problem (i.e., problems with zero right-hand sides $F=0$ and $f=0$ ) is finite-dimensional and there exists a finite-dimensional subspace $\widetilde{X} \subseteq C^{\mu}(\bar{D}) \times C^{\mu}(\Gamma)$ such that the orthogonality conditions

$$
\langle(F, f),(\widetilde{F}, \widetilde{f})\rangle=0, \quad(\widetilde{F}, \widetilde{f}) \in \widetilde{X},
$$

are necessary and sufficient for the solvability of the inhomogeneous problem. The difference

$$
\varkappa=\operatorname{dim} X-\operatorname{dim} \tilde{X}
$$

determines the index of the problem.

Theorem 1. Let $\Gamma \in C^{1, \nu}$ and $C \in C^{\nu}(\Gamma), \mu<\nu<1$, so that the matrix-valued function $G(t)=$ $\left(C(t) B_{1}, \overline{C(t)} B_{2}\right)$ (see the notation $(3)$ ) belongs to the class $C^{\nu}(\Gamma)$. Then the condition

$$
\operatorname{det} G(t) \neq 0, \quad t \in \Gamma,
$$

holds if and only if the problem (1), (4) be is a Fredholm problem in the class $C^{\mu}(\bar{D}) \cap C^{1}(D)$, and its index is given by the formula

$$
\varkappa=-\sum_{j=1}^{m} \frac{1}{\pi}[\arg \operatorname{det} G]_{\Gamma_{j}}+(2-m) l,
$$

where $\Gamma_{1}, \ldots, \Gamma_{m}$ are simple contours composing $\Gamma$ and the increment []$_{\Gamma_{j}}$ along $\Gamma_{j}$ is taken in the direction of leaving the domain $D$ to the left.

If, in addition, $C \in C^{1, \nu}(\Gamma)$, then any solution $U \in C^{\mu}(\bar{D}) \cap C^{1}(D)$ of the problem with the righthand side $f \in C^{1, \mu}(\Gamma)$ actually belongs to $C^{1, \mu}(\bar{D})$.

Proof. According to (2), we write the vector-valued function $\widetilde{\phi}=\widetilde{B}^{-1} U$ as a pair $\left(\phi_{1}, \bar{\phi}_{2}\right)$ with $l_{k}$-vectors $\phi_{k}$. After this substitution, (1) turns into the system

$$
\frac{\partial \widetilde{\phi}}{\partial y}-\widetilde{J} \frac{\partial \widetilde{\phi}}{\partial x}+\widetilde{a}(z) \widetilde{\phi}(z)+\widetilde{b}(z) \overline{\widetilde{\phi}(z)}=\widetilde{f}^{1}(z)
$$

with the coefficients $\widetilde{a}=\widetilde{B}^{-1} a \widetilde{B}$ and $\widetilde{b}=\widetilde{B}^{-1} b \widetilde{B}$ and the right-hand side $\widetilde{f}^{1}=\widetilde{B}^{-1} F$. We rewrite it in the componentwise block form:

$$
\begin{aligned}
& \frac{\partial \phi_{1}}{\partial y}-J_{1} \frac{\partial \phi_{1}}{\partial x}+a_{11} \phi_{1}+a_{12} \bar{\phi}_{2}+b_{11} \bar{\phi}_{1}+b_{12} \phi_{2}=f_{1}^{1}, \\
& \frac{\partial \bar{\phi}_{2}}{\partial y}-\bar{J}_{2} \frac{\partial \bar{\phi}_{2}}{\partial x}+a_{21} \bar{\phi}_{1}+a_{22} \phi_{2}+b_{21} \phi_{1}+b_{22} \bar{\phi}_{2}=\bar{f}_{2}^{1},
\end{aligned}
$$


where $\left(a_{i j}\right)=\widetilde{a}$ and $\left(b_{i j}\right)=\widetilde{b}$ are block matrices and $\left(f_{1}^{1}, \bar{f}_{2}^{1}\right)=\widetilde{f}^{1}$. Replacing the second equation of this system by its complex conjugate, we obtain the following system for the vector $\phi=\left(\phi_{1}, \phi_{2}\right)$ :

$$
\frac{\partial \phi}{\partial y}-J \frac{\partial \phi}{\partial x}+c \phi+d \bar{\phi}=f^{1}
$$

where $J=\operatorname{diag}\left(J_{1}, J_{2}\right)$ is a block-diagonal matrix, the right-hand side $f^{1}=\left(f_{1}^{1}, f_{2}^{1}\right) \in C^{\mu}(\bar{D})$, and the coefficients have the form

$$
c=\left(\begin{array}{ll}
a_{11} & b_{12} \\
b_{21} & a_{22}
\end{array}\right), \quad d=\left(\begin{array}{cc}
b_{11} & a_{12} \\
a_{21} & b_{22}
\end{array}\right) \in C^{\mu}(\bar{D}) .
$$

Since $U=\widetilde{B} \widetilde{\phi}=B_{1} \phi_{1}+\bar{B}_{2} \bar{\phi}_{2}$, after this substitution the boundary condition (4) takes the form

$$
\operatorname{Re} G \phi^{+}=f^{0},
$$

where for uniformity with (8) we denote the function $f$ by $f^{0}$.

The problem (8), (9) for this system (under the assumption that $\Gamma$ is a simple contour) is studied in [10]. The corresponding arguments of that paper with minor changes are also suitable for the considered case of a composite contour. Keeping in mind the smoothness of solutions to the problem, we briefly recall these arguments.

Using the matrix notation $z_{J}=x \cdot 1+y \cdot J$, for $z=x+i y \in \mathbb{C}$, we introduce the following Cauchy-type integral operator:

$$
\left(I^{0} \varphi\right)(z)=\frac{1}{2 \pi i} \int_{\Gamma}(t-z)_{J}^{-1} d t_{J} \varphi(t), \quad z \in D,
$$

where $t=t_{1}+i t_{2}$ is a point on the contour $\Gamma$, which is oriented positively with respect to $D, d t_{J}$ denotes the complex matrix differential $d t_{1} 1+d t_{2} J$, and the singular Cauchy operator

$$
\left(S^{0} \varphi\right)\left(t_{0}\right)=\frac{1}{\pi i} \int_{\Gamma}\left(t-t_{0}\right)_{J}^{-1} d t_{J} \varphi(t), \quad t_{0} \in \Gamma,
$$

where $\varphi \in C^{\mu}(\Gamma)$ is a real $l$-vector-valued function.

Following [7], we say that the function $\phi=I^{0} \psi$ is $J$-analytic in the domain $D$, i.e., it satisfies the equation

$$
\frac{\partial \phi}{\partial y}-J \frac{\partial \phi}{\partial x}=0
$$

For $J=i$, this system becomes the classical Cauchy-Riemann system. As was shown in [7], all basic facts of the theory of analytic functions associated with the integral Cauchy formula can be extended to solutions of the system (14).

According to [6], the integral operator $I^{0}: C^{\mu}(\Gamma) \rightarrow C^{\mu}(\bar{D})$ is bounded and the following SokhotskPlemelj formula is valid:

$$
2\left(I^{0} \varphi\right)^{+}\left(t_{0}\right)=\varphi\left(t_{0}\right)+\left(S^{0} \varphi\right)\left(t_{0}\right), \quad t_{0} \in \Gamma .
$$

Obviously, in the case of the scalar matrix $J=i$, the operator $S^{0}$ becomes a classical singular Cauchy operator, which we denote by $S$. As was shown in [6], under the assumption $\Gamma \in C^{1, \nu}$, the difference $S^{0}-S$ is a compact operator in the space $C^{\mu}(\Gamma)$, and all principal results of the classical theory of singular operators (see [4]) can also be applied to the operator

$$
2 N^{0} \varphi=\operatorname{Re}\left[G\left(\varphi+S^{0} \varphi\right)\right]
$$

acting in the space of real $l$-vector-valued functions $\varphi \in C^{\mu}(\Gamma)$.

Thus, this operator is a Fredholm operator if and only if the condition (6) is satisfied, and its index is given by the formula

$$
\operatorname{ind} N^{0}=-\left.\frac{1}{\pi}[\arg \operatorname{det} G]\right|_{\Gamma^{\circ}}
$$


Now we introduce integral operators in the domain

$$
\begin{aligned}
& \left(I^{1} \varphi\right)(z)=\frac{1}{\pi i} \int_{D}(t-z)_{J}^{-1} \varphi(t) d_{2} t, \quad z \in D, \\
& \left(S^{1} \varphi\right)(z)=\frac{1}{\pi i} \int_{D}(t-z)_{J}^{-2} \varphi(t) d_{2} t, \quad z \in D .
\end{aligned}
$$

The last integral is singular and is understood in the corresponding sense. It is easy to verify the following necessary condition for the existence of this integral:

$$
\int_{\mathbb{T}} \xi_{J}^{-2} d_{1} \xi=0,
$$

where $\mathbb{T}$ is the unit circle. We note that, due to the evenness of the function $\xi_{J}^{-2}$, the relation

$$
\int_{\mathbb{T}^{+}} \xi_{J}^{-2} d_{1} \xi=0
$$

also holds, where $\mathbb{T}^{+}$means any semicircle.

As was shown in [11], for $\varphi \in C^{\mu}(\bar{D})$ the function $I^{1} \varphi$ is continuously differentiable in the domain $D$ and the following formulas are valid:

$$
\frac{\partial\left(I^{1} \varphi\right)}{\partial x}=\sigma_{1} \varphi+S^{1} \varphi, \quad \frac{\partial\left(I^{1} \varphi\right)}{\partial y}=\sigma_{2} \varphi+J S^{1} \varphi
$$

where $\sigma_{k} \in \mathbb{C}^{l \times l}$ are certain matrices related by the equation $\sigma_{2}=J \sigma_{1}$. In particular,

$$
\left(\frac{\partial}{\partial y}-J \frac{\partial}{\partial x}\right) I^{1} \varphi=0
$$

By (14), we can apply [9, Theorem 3.5.1] to the singular integral operator $S^{1}$. According to this theorem, this operator is bounded in $C^{\mu}(\bar{D})$. Taking into account (15), we conclude that the operator $I^{1}: C^{\mu}(\bar{D}) \rightarrow C^{1, \mu}(\bar{D})$ is bounded.

We consider the functional class

$$
\phi \in C^{\mu}(\bar{D}) \cap C^{1}(D), \quad\left(\frac{\partial}{\partial y}-J \frac{\partial}{\partial x}\right) \phi \in C^{\mu}(\bar{D}) .
$$

Obviously, any solution $\phi \in C^{\mu}(\bar{D}) \cap C^{1}(D)$ of Eq. (8) automatically belongs to this class.

For definiteness, we assume that the contour $\Gamma_{m}$ encircles the remaining contours $\Gamma_{1}, \ldots, \Gamma_{m-1}$. Then any function $\phi \in C^{\mu}(\bar{D})$ can be uniquely represented in the form

$$
\phi=I^{1} \varphi^{1}+I^{0} \varphi^{0}+i \xi, \quad \xi \in \mathbb{R}^{l}
$$

with some complex $l$-vector-valued function $\varphi^{0} \in C^{\mu}(\Gamma)$ and a real vector-valued function $\varphi^{0} \in C^{\mu}(\Gamma)$ satisfying the conditions

$$
\int_{\Gamma_{j}} \varphi(t) d_{1} t=0, \quad 1 \leq j \leq m-1 .
$$

In fact, we assume

$$
\varphi^{1}=\left(\frac{\partial}{\partial y}-J \frac{\partial}{\partial x}\right) \phi
$$

and let $\phi^{0}=\phi-I^{1} \varphi^{1}$. Then, due to (16), the function $\phi^{0}$ is $J$-analytic in the domain $D$, i.e., it satisfies Eq. (10) and belongs to the class $C^{1, \mu}(\bar{D})$. Therefore, the problem is reduced to the representation

$$
\phi^{0}=I^{0} \varphi^{0}+i \xi, \quad \xi \in \mathbb{R}^{l}
$$


under the conditions (19) on the real density $\varphi$, which is established in [6] (see also [8]).

Using the representation (18) and the Sokhotski-Plemelj formula (11), we can reduce the problem (8), (9) to the following equivalent system of operator equations:

$$
N^{1} \varphi^{1}+N^{10} \varphi^{0}+i(c-d) \xi=f^{1}, \quad N^{01} \varphi^{1}+N^{0} \varphi^{0}-(\operatorname{Im} G) \xi=f^{0},
$$

where, in addition to (12), for brevity we introduce the notation

$$
\begin{gathered}
N^{1} \varphi^{1}=\varphi^{1}+c\left(I^{1} \varphi^{1}\right)+d\left(\overline{I^{1} \varphi^{1}}\right), \\
N^{10} \varphi^{0}=c\left(I^{1} \varphi^{0}\right)+d\left(\overline{I^{1} \varphi^{0}}\right), \quad N^{01} \varphi^{1}=\operatorname{Re} G\left(I^{1} \varphi^{1}\right)^{+} .
\end{gathered}
$$

This system is considered with respect to the set $\left(\varphi^{1}, \varphi^{0}, \xi\right)$, subject to the conditions (19). We can write it briefly using the notation $\varphi=\left(\varphi^{1}, \varphi^{0}\right)$ :

$$
N \varphi+T \xi=f
$$

with the right-hand side $f=\left(f^{1}, f^{0}\right)$ and the operator matrices

$$
N=\left(\begin{array}{cc}
N^{1} & N^{10} \\
N^{01} & N^{0}
\end{array}\right), \quad T=\left(\begin{array}{c}
i c-i d \\
-\operatorname{Im} G
\end{array}\right) .
$$

Obviously, the space $C^{\mu}(\bar{D}) \times C^{\mu}(\Gamma) \times \mathbb{R}^{l}$ is an extension of the space $C^{\mu}(\bar{D}) \times C^{\mu}(\Gamma)$ to $l$ dimensions; therefore, based on well-known properties of Fredholm operators (see [5]), we conclude that the operators $(N, T)$ and $N$ are Fredholm equivalent and their indices are related by the formula

$$
\operatorname{ind}(N, T)=\operatorname{ind} N+l \text {. }
$$

On the other hand, the condition (19) determines a closed subspace of codimension $l(m-1)$ in the space $C^{\mu}(\Gamma)$; therefore, from the same considerations, the index $\varkappa$ of the system (19), (20) is related to the index of the operator $(N, T)$ by the formula

$$
\varkappa=\operatorname{ind}(N, T)-l(m-1) .
$$

Let us consider in detail the operators appeared in (20). We will write $N_{1} \sim N_{2}$ if the difference $N_{1}-N_{2}$ is a compact operator. Recall that the operator $I^{1}$ is compact in $C^{\mu}(\bar{D})$; then we can write $N^{1} \sim 1, N^{01} \sim 0$, and consequently,

$$
N \sim M=\left(\begin{array}{cc}
1 & N^{1,0} \\
0 & N^{0}
\end{array}\right)
$$

Assume that the condition (6) is satisfied. Then, as was noted above, the operator $N^{0}$ is a Fredholm operator and its index is given by the formula (13). In particular, there exists its regularizer, i.e. an operator $R^{0}$ in $C^{\mu}(\Gamma)$ possessing the property $R^{0} N^{0} \sim N^{0} R^{0} \sim 1$. One can directly verify that the operator

$$
R=\left(\begin{array}{cc}
1 & -N^{1,0} R^{0} \\
0 & R^{0}
\end{array}\right)
$$

is a regularizer of the operator $M$ and hence the operator $M$ is a Fredholm operator. This implies that the operator $N$ is a Fredholm operator and hence the initial problem (1), (4) is a Fredholm problem.

Conversely, let the problem (1), (4) be a Fredholm problem such that $N$ and hence $M$ are Fredholm operators. Let $R$ be the regularizer written in the block form:

$$
R=\left(\begin{array}{cc}
R^{1} & R^{10} \\
R^{01} & R^{0}
\end{array}\right)
$$

Then it follows directly from the relations $M R \sim M R \sim 1$ that $N^{0} R^{0} \sim R^{0} N^{0} \sim 1$, so that $N^{0}$ is a Fredholm operator. As was noted above, this implies the condition (6).

In order to prove the formula (7) for the index, we introduce the operator $M(t)$ depending on the parameter $0 \leq t \leq 1$, which is obtained by replacing of $N^{10}$ by $t N^{10}$ in the definition (24) of the 
operator $M$. The same arguments show that $M(t)$ is also a Fredholm operator. Since it depends on $t$ continuously, its index is independent of $t$ and, in particular,

$$
\text { ind } M=\operatorname{ind} M(0)=\operatorname{ind} N^{0} \text {. }
$$

Hence ind $N=$ ind $N^{0}$, which together with (13), (22), and (23) completes the proof of the index formula (7).

Now we turn to the last assertion of the theorem and assume that the functions $C$ and hence $G$ also belong to $C^{1, \mu}(\Gamma)$. Then for $f^{0} \in C^{1, \mu}(\Gamma)$, the terms $N^{01} \varphi^{1}$ and $(\operatorname{Im} G) \xi$ in the second equation $(20)$ also belong to $C^{1, \mu}(\Gamma)$. Thus, the function $\varphi^{0} \in C^{\mu}(\Gamma)$ satisfies the equation $N^{0} \varphi^{0}=g$ with the righthand side $g \in C^{1, \mu}(\Gamma)$. As was shown in [1], in this case $\varphi^{0} \in C^{1, \mu}(\Gamma)$. According to the differentiation formula for the Cauchy-type integral $\phi=I^{0} \varphi$ (see [9]), the function $I^{0} \varphi^{0}$ belongs to $C^{1, \mu}(\bar{D})$, so that the function $\phi$ in the representation (18) belongs to this class, and hence the solution $U=B_{1} \phi_{1}+B_{2} \phi_{2}$ of the original problem (1), (4) also belongs to this class.

Note that due to the last assertion of the theorem, the problem (1), (4) is a Fredholm problem in the class $C^{1, \mu}(\bar{D})$ with the same index.

The case of the elliptic system

$$
\frac{\partial U}{\partial y}-A \frac{\partial U}{\partial x}+a(z) U(z)=F(z)
$$

with real coefficients $A \in \mathbb{R}^{2 l \times 2 l}$ and $a(z)$ can be considered similarly. In this case, eigenvalues of the matrix $A$ form the set $\sigma \cup \bar{\sigma}$, where $\sigma \subseteq\{\nu, \operatorname{Im} \nu>0\}$. Therefore, the relations (2) and (3) take the form

$$
\widetilde{B}^{-1} A \widetilde{B}=\widetilde{J}, \quad \widetilde{J}=\operatorname{diag}(J, \bar{J}), \quad \widetilde{B}=(B, \bar{B}), \quad B \in \mathbb{C}^{2 l \times l} .
$$

Here the boundary condition

$$
C U^{+}=f
$$

with a $(l \times 2 l)$-matrix $C(t), t \in \Gamma$, is an analog of the Riemann-Hilbert problem; respectively, the following assertion (with the same proof) is an analog of Theorem 1.

Theorem 2. Let $\Gamma \in C^{1, \nu}$ and an $(l \times 2 l)$-matrix $C$ belong to the class $C^{\nu}(\Gamma), \mu<\nu<1$, so that we have $G(t)=C(t) B \in C^{\nu}(\Gamma)$ (see the notation (3). The problem (25), (26) is a Fredholm problem in the class $C^{\mu}(\bar{D}) \cap C^{1}(D)$ if and only if the condition (6) holds, and its index is given by the formula (7).

If, in addition, $C \in C^{1, \nu}(\Gamma)$, then any solution $U \in C^{\mu}(\bar{D}) \cap C^{1}(D)$ of the problem with the righthand side $f \in C^{1, \mu}(\Gamma)$ actually belongs to $C^{1, \mu}(\bar{D})$.

We note that in a few more general classes, Theorem 2 was established by B. Bojarski in [2] (see also [3]).

Up to now, the domain $D$ has been assumed to be finite, i.e., lying inside a certain circle. Now we consider the case where the domain $D$ is still bounded by a contour $\Gamma \in C^{1, \nu}$ but is infinite, i.e., it contains the outer domain of a certain circle. In this case, all simple contours $\Gamma_{j}, 1 \leq j \leq m$, which form the contour $\Gamma$, are equivalent. For simplicity, we assume that the point $z=0$ lies outside $\bar{D}$.

We perform our considerations in the weighted Hölder space $C_{\delta}^{\mu}(\bar{D}, \infty), \delta \in \mathbb{R}$, with power behavior $O\left(|z|^{\delta}\right)$ at infinity (see [9]). We briefly recall its definition. The space $C_{0}^{\mu}(\bar{D}, \infty)$ consists of all bounded functions $\varphi(z), z \in D$ with finite norm $|\varphi|=|\varphi|_{0}+\{\varphi\}_{\mu}$, where

$$
|\varphi|_{0}=\sup _{z \in D}|\varphi(z)|, \quad\{\varphi\}_{\mu}=\sup _{z_{1} \neq z_{2}} \frac{\left|z_{1}\right|^{\mu}\left|\varphi\left(z_{1}\right)-\varphi\left(z_{2}\right)\right|}{\left|z_{1}-z_{2}\right|^{\mu}} .
$$

This space is a Banach algebra with respect to multiplication, and the weighted space $C_{\delta}^{\mu}$ is obtained from $C_{0}^{\mu}$ by multiplying its elements by $|z|^{\delta}$ (with the transferred norm). By definition, the 
space $C_{\delta}^{1, \mu}(\bar{D}, \infty)$ consists of functions $\varphi \in C_{\delta}^{\mu}(\bar{D}, \infty) \cap C^{1}(D)$ whose partial derivatives belong to the class $C_{\delta-1}^{\mu}(\bar{D}, \infty)$ :

$$
\frac{\partial \varphi}{\partial x}, \frac{\partial \varphi}{\partial y} \in C_{\delta-1}^{\mu}(\bar{D}, \infty)
$$

We consider Eq. (1) in the infinite domain $D$ in the class

$$
C_{\lambda}^{\mu}(\bar{D}, \infty) \cap C^{1}(D), \quad-1<\lambda<0,
$$

assuming that

with some $\varepsilon>0$.

$$
a, b \in C_{-1-\varepsilon}^{\mu}(\bar{D}, \infty), \quad F \in C_{\lambda-1}^{\mu}(\bar{D}, \infty)
$$

Consider the operators $I^{0}$ and $I^{1}$ introduced above. In the case considered, the function $\left(I^{0} \varphi\right)(z)$ has the following expansion in a neighborhood of $\infty$ :

$$
\left(I^{0} \varphi\right)(z)=\sum_{k \leq-1} c_{k} z_{J}^{k}, \quad c_{k}=-\frac{1}{2 \pi i} \int_{\Gamma} t_{J}^{-k-1} d t_{j} \varphi(t),
$$

and since $-1<\lambda<0$, the operator $I^{0} H C^{\mu}(\Gamma) \rightarrow C_{\lambda}^{\mu}(\bar{D}, \infty)$ is bounded. We state the corresponding properties of the operator $I^{1}$.

Theorem 3. The operator $I^{1}$ considered as an operator $C_{\lambda-1}^{\mu}(\bar{D}, \infty) \rightarrow C_{\lambda}^{\mu}(\bar{D}, \infty)$ is bounded and being considered as an operator $C_{\lambda-1}^{\mu}(\bar{D}, \infty) \rightarrow C_{\lambda+\varepsilon}^{\mu}(\bar{D}, \infty), \varepsilon>0$, it is compact. Moreover, any function $\phi$ of the class

$$
\phi \in C_{\lambda}^{\mu}(\bar{D}) \cap C^{1}(D), \quad\left(\frac{\partial}{\partial y}-J \frac{\partial}{\partial x}\right) \phi \in C_{\lambda-1}^{\mu}(\bar{D}),
$$

can be uniquely represented in the form

$$
\phi=I^{0} \varphi^{0}+I^{1} \varphi^{1}
$$

with a real vector-valued function $\varphi^{0} \in C^{\mu}(\Gamma)$ satisfying the condition

$$
\int_{\Gamma_{j}} \varphi(t) d_{1} t=0, \quad 1 \leq j \leq m,
$$

and a complex vector-valued function $\varphi^{1} \in C_{\lambda-1}^{\mu}(\bar{D}, \infty)$.

Proof. The first statement of the theorem on the boundedness of the operator $I^{1}: C_{\lambda-1}^{\mu}(\bar{D}, \infty) \rightarrow$ $C_{\lambda}^{\mu}(\bar{D}, \infty)$ is established similarly to [9]. It was also established in [9] that the singular operator $S^{1}$ is bounded in the space $C_{\lambda-1}^{\mu}(\bar{D}, \infty)$. Hence, taking into account (16) and the compactness property of the embedding mentioned in [9],

$$
C_{\delta-\varepsilon}^{\mu+\varepsilon}(\bar{D}, \infty) \subseteq C_{\delta}^{\mu}(\bar{D}, \infty), \quad \varepsilon>0,
$$

we conclude that the operator $I^{1}$ is compact.

The second part of the theorem is established similarly to the case of a finite domain.

Using Theorem 3 and following the scheme of the proof of Theorem 1, we arrive at the validity of the following assertion.

Theorem 4. Assume that a contour $\Gamma$ belongs to the class $C^{1, \nu}, C$ belongs to the class $C^{\nu}(\Gamma), \mu<$ $\nu<1$, and $G=\left(C B_{1}, \bar{C} B_{2}\right)$. Then under the assumption (28), the problem (1), (4) is a Fredholm problem in the class (27) if and only if the condition (6) holds. Moreover, its index is given by the formula

$$
\varkappa=-\sum_{j=1}^{m} \frac{1}{\pi}[\arg \operatorname{det} G]_{\Gamma_{j}}-m l .
$$


If, in addition, $C \in C^{1, \nu}(\Gamma)$, then any solution $U$ of the problem of this class with right-hand side $f \in C^{1, \mu}(\Gamma)$ in fact belongs to $C_{\lambda}^{1, \mu}(\bar{D}, \infty)$.

An analog of Theorem 2 for the system (1) with real coefficients can be formulated similarly.

Acknowledgment. This work was supported by the Ministry of Education and Science of the Russian Federation (project No. 1.7311.2017/BCh).

\section{REFERENCES}

1. E. A. Abapolova and A. P. Soldatov, "On the theory of singular integral equations on a smooth contour," Nauchn. Vedom. Belgorod. Univ., No. 5 (76), issue 18, 6-20 (2010).

2. B. V. Bojarski, "Theory of generalized analytic vectors," Ann. Polon. Math., 17, No. 3, 281-320 (1965).

3. R. P. Gilbert and J. L. Buchanan, First-Order Elliptic Systems: A Function Theoretic Approach, Academic Press, New York (1983).

4. N. I. Muskhelishvili, Singular Integral Equations [in Russian], Nauka, Moscow (1968).

5. R. S. Palais, Seminar on the Atiyah-Singer Index Theorem, Ann. Math. Stud., 57, Princeton Univ. Press, Princeton, New Jersey (1965).

6. A. P. Soldatov, "A function theory method in boundary-value problems in the plane. I. The smooth case," Izv. Akad. Nauk SSSR. Ser. Mat., 55, No. 5, 1070-1100 (1991).

7. A. P. Soldatov, "Hyperanalytic functions and their applications," J. Math. Sci., 132, No. 6, 827-882 (2006).

8. A. P. Soldatov, "The Schwarz problem for Douglis analytic functions," J. Math. Sci., 173, No. 2, 221-224 (2011).

9. A. P. Soldatov, "Singular integral operators and elliptic boundary-value problems. I," Sovr. Mat. Fundam. Napr., 63, No. 1, 1-189 (2017).

10. A. P. Soldatov and O. V. Chernova, "The Riemann-Hilbert problem for a first-order elliptic system in Hölder classes," Nauch. Vedom. Belgorod. Univ., No. 13 (68), issue 17/2, 115-121 (2009).

11. O. V. Vashchenko and A. P. Soldatov, "Integral representation of solutions of the generalized Beltrami system," Nauchn. Vedom. Belgorod. Univ. Ser. Inform. Prikl. Mat., No. 6, issue 1 (21), 3-6 (2006).

A. P. Soldatov

Federal Research Center "Information and Management,"

Russian Academy of Sciences, Moscow, Russia

E-mail: soldatov48@gmail.com

O. V. Chernova

Belgorod National Research University, Belgorod, Russia

E-mail: volga@mail.ru 